\title{
Self-Calibration of a Moving Camera by Pre-Calibration
}

\author{
Peter Sturm \\ GRAVIR-IMAG \& INRIA Rhône-Alpes* \\ 655, Avenue de l'Europe, 38330 Montbonnot, France \\ Peter.Sturmeinrialpes.fr
}

\begin{abstract}
We consider the problem of self-calibrating a moving camera which is equipped with a zoom lens. This consists essentially in estimating the 5 intrinsic parameters of the pinhole camera model. However, these parameters are not independent. Thus, we propose to do a pre-calibration of the camera, with the aim to model the interdependence of the intrinsic parameters. We show that self-calibration then comes down to the estimation of only 1 intrinsic parameter. We propose a method which exploits this and which does not need an initialization of the intrinsic parameters.
\end{abstract}

\section{Introduction}

In this paper we address the problem of self-calibration of a moving camera. This means essentially the determination of the camera's intrinsic parameters, only from the information of image point correspondences. In the pioneering work of Maybank and Faugeras ([1]), the authors consider constraints on the intrinsic parameters from rigid motion, based on the epipolar geometry of two views. These constraints are known as Kruppa's equations. Experimentations with methods based on these equations reveal imprecision and inaccuracy due to high sensitivity to noise, and also convergence problems ([2]).

To moderate these effects, it is proposed to reduce the number of unknowns by fixing some of the intrinsic parameters to predefined values $([2,3,4])$. While there is no problem for fixing the aspect ratio and the skew of the pixel coordinate axes, which are very stable over long time periods, the position of the principal point depends on the zooming position and lens focus of the camera ([5, 6]) (see Figure 1). This phenomenon is due to optical and mechanical misalignments in the lens system of a camera and can occur in such an extreme manner as shown in Figure 4, where the coordinates of the principal point vary up to 100 pixels while zooming !

On one hand, this illustrates that fixing the position of the principal point can bias heavily the results of self-calibration. On the other hand, although the intrinsic parameters are correlated, the correlation seems to be simple and stable.

This led to the idea that, if it is possible to obtain a simple analytical model which approximates the behavior of intrinsic parameters as a function of other parameters, this

\footnotetext{
${ }^{*}$ This work has been done in the context of the Movi project which belongs to CNRS, INPG, INRIA and UJF.
} 


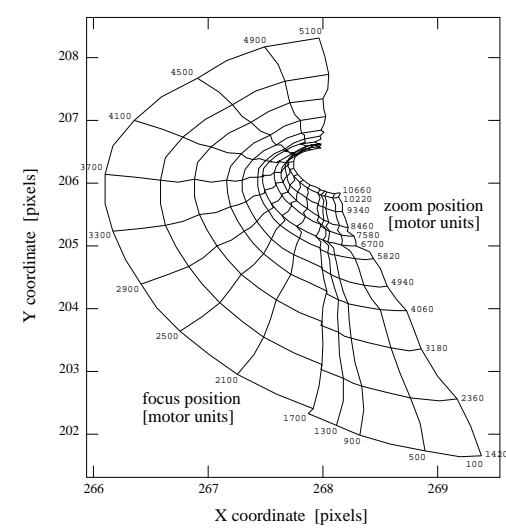

Figure 1: Image center as a function of lens focus and zoom motors ([5]).

knowledge could be introduced into any self-calibration method in order to reduce the number of unknowns and to improve the accuracy of the results. Thus, we want to model the interdependence of intrinsic parameters, concretely between the position of the principal point and the horizontal and vertical scale factors.

We propose to precede the application of a dynamic vision system by a pre-calibration stage, where a model for the interdependence of intrinsic parameters is established. We show, that dynamic self-calibration may then be reduced to the estimation of one parameter, on which the other parameters depend. The advantages of this approach are a simpler and faster computation, higher accuracy and no need of an initial estimation, thanks to the off-line elimination of correlation.

The paper is organized as follows. (1) We discuss the pinhole camera model and especially several aspects of the intrinsic parameters. (2) Kruppa's equations are derived and it is shown how to use them for self-calibration. (3) We show how information on the interdependence of intrinsic parameters can be introduced in the self-calibration process. (4) Based on this consideration, we propose a self-calibration method, which exploits this information. (5) Results of experiments with the self-calibration method are reported. (6) Finally, we discuss our observations and propositions and indicate how this work can be extended.

\section{Intrinsic Parameters of a Zooming Camera}

\subsection{The Pinhole Model}

In the following we model cameras by the pinhole scheme which is a good approximation to the physical reality. A camera is represented by a projection center $\boldsymbol{O}$ and a retinal plane $\mathcal{R}$. The projection $\boldsymbol{q}$ of a 3D point $\boldsymbol{Q}$ is the intersection of the line $\langle\boldsymbol{O}, \boldsymbol{Q}\rangle$, with the retinal plane. This projection can be represented by a $3 \times 4$ projection matrix $\boldsymbol{P}$ such that $\boldsymbol{q} \approx \boldsymbol{P Q}$, where $\boldsymbol{Q}$ and $\boldsymbol{q}$ are represented by homogeneous coordinates and $\approx$ means equality up to a scalar factor. The optical axis is the line through the projection center and perpendicular to the retinal plane. Its intersection with the retinal plane is the principal point. 


\subsection{Extrinsic and Intrinsic Parameters}

We can identify two groups of physically meaningful parameters which describe entirely the pinhole projection: 6 extrinsic parameters stand for the position and orientation of the camera, and 5 intrinsic parameters characterize the camera's projection properties. The intrinsic parameters are :

- $u_{0}$ and $v_{0}$, the coordinates of the principal point.

- $\alpha_{u}$ and $\alpha_{v}$, the horizontal and vertical scale factors.

- $\theta$, the skew angle between the coordinate axes in the pixel coordinate system.

The projection matrix can be decomposed as

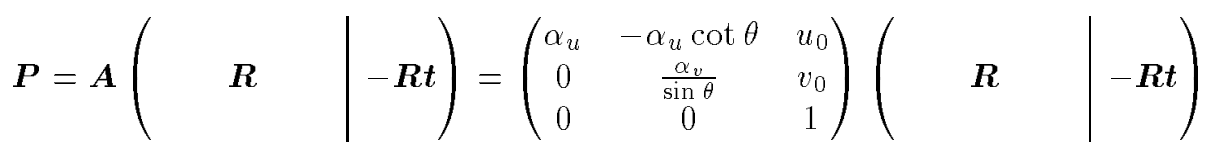

where $\boldsymbol{A}$ is the calibration matrix of the camera, $\boldsymbol{t}$ the position of the projection center and the orthonormal matrix $\boldsymbol{R}$ represents the camera's orientation.

\subsection{The Absolute Conic and the Intrinsic Parameters}

Let $\Omega$ be the absolute conic, i.e. the conic which is formed by the points $(x, y, z, w)^{T}$ $\in \mathcal{P}^{3}$ with $x^{2}+y^{2}+z^{2}=0$ and $w=0$ ([7]). An interesting property of this conic is that its projection $\omega$ depends only on the intrinsic parameters of a camera, i.e. $\omega$ is fixed for a moving camera with fixed intrinsic parameters. The projection of the absolute conic by a camera with calibration matrix $\boldsymbol{A}$ is given by $C^{*} \approx \boldsymbol{A}^{T}$ ([2]), where $C$ is the matrix representation of $\omega$ and $C^{*}$ denotes its dual matrix ${ }^{1}$. Determination of the intrinsic parameters is equivalent to determination of the projection of the absolute conic, since $\boldsymbol{A}$ may be uniquely computed from $\boldsymbol{C}$ by Choleski decomposition. This principle is used for self-calibration via Kruppa's equations (see 3.2).

\subsection{Stability of the Intrinsic Parameters}

Some of the intrinsic parameters are constant over long time periods. Especially, the aspect ratio $\tau=\frac{\alpha_{u}}{\alpha_{2}}$ and the skew angle $\theta$ do not change in general. In practice $\theta$ is often very close to $90^{\circ}$. So, in many works concerning calibration or self-calibration, $\theta$ is assumed to be $90^{\circ}$ and is not estimated.

As for the principal point, it has been shown that it is not stable, rather its position varies when the zoom position and the lens focus change ([5, 6]). These authors have observed that zooming causes the principal point to move on a nearly translational trajectory (for most zoom objectives). However, in the following we will not restrict ourselves to the case of a translation, rather we consider a generic model. The reasons for this phenomenon are optical and mechanical misalignments in the lens system of a camera.

In close-range applications of computer vision, the range of useful focus positions is small. Hence, in the following we will suppose that the influence on the intrinsic parameters comes mainly from the zoom position and neglect the effects of changing the focus.

\footnotetext{
${ }^{1}$ The dual to a conic $\mathcal{C}$ of points is the conic which consists of the tangent lines of $\mathcal{C}$
} 


\subsection{Interdependence of Intrinsic Parameters}

Despite the revelation of the influence of zooming on the parameters $\alpha_{u}, \alpha_{v}, u_{0}$ and $v_{0}$, the interdependence of these parameters, subject to zooming, has to our knowledge not yet been used in the context of self-calibration. We want to profit from these interdependencies and try therefore to obtain an analytical model which expresses the intrinsic parameters as functions of one of them, say $\alpha_{v}$ (the skew angle $\theta$ is considered to be fix) :

$$
\boldsymbol{A}=\left(\begin{array}{ccc}
\alpha_{u}\left(\alpha_{v}\right) & -\alpha_{u}\left(\alpha_{v}\right) \cot \theta & u_{0}\left(\alpha_{v}\right) \\
0 & \frac{\alpha_{v}}{\sin \theta} & v_{0}\left(\alpha_{v}\right) \\
0 & 0 & 1
\end{array}\right)
$$

For the horizontal scale factor we always have the relation $\alpha_{u}=\tau \frac{\alpha_{v}}{\sin \theta}$, whereas the functional relationships $u_{0}\left(\alpha_{v}\right)$ and $v_{0}\left(\alpha_{v}\right)$ are more difficult to model.

In the following we call pre-calibration of a camera the off-line determination of the aspect ratio $\tau$, the functional relationships $u_{0}\left(\alpha_{v}\right)$ and $v_{0}\left(\alpha_{v}\right)$, and the skew angle $\theta$. If a camera is pre-calibrated, self-calibration will therefore be reduced to the estimation of the sole parameter $\alpha_{v}$, and it will be possible to develop special methods exploiting this. We will discuss this in sections 4 and 5 .

\section{Self-Calibration of a Moving Camera}

\subsection{Problem Specification}

By self-calibration we mean the estimation of the intrinsic parameters of a camera, by just pointing it at a rigid scene. Especially, no 3D model of the scene and no information on the camera motion are available. The only information needed are correspondences of image points (or other features).

In this paper, we adopt the self-calibration approach which is based on Kruppa's equations, however the remarks on self-calibration of a pre-calibrated camera in section 5 are also valid for other paradigms ([8, 9]).

In the sequel, we suppose the epipolar geometry of pairs of views known (it can be computed from point matches). We use its representation by the fundamental matrix $\boldsymbol{F}$ ([10]) which includes the position of the epipoles $e$ and $e^{\prime}$.

\subsection{Kruppa's Equations and Self-Calibration}

The classical (though recent) self-calibration approach in the computer vision domain is based on Kruppa's equations ([1]). If we assume that the intrinsic parameters are the same for two positions of one camera, then constraints on the projection of the absolute conic can be derived from the epipolar geometry of the two positions. These constraints can be expressed in the form

$$
c_{2} c_{1}^{\prime}=c_{1} c_{2}^{\prime} \quad c_{2} c_{0}^{\prime}=c_{0} c_{2}^{\prime} \quad c_{1} c_{0}^{\prime}=c_{0} c_{1}^{\prime} .
$$

where the coefficients $c_{i}$ and $c_{i}^{\prime}$ depend on $\boldsymbol{F}, e$ and $\boldsymbol{K}$ ([1]). These three equations (only two of them are algebraically independent) are the Kruppa equations. 
When the epipolar geometry, i.e. $\boldsymbol{F}$ and $\boldsymbol{e}$, is known, the equations are constraints on $\boldsymbol{K}$ and hence on the intrinsic parameters. Thus they can be used for self-calibration. The usual approach of self-calibration consists in minimizing the criterion ([2])

$$
\sum_{p=1}^{\text {\#pairs of views }}\left(\frac{c_{p 2}}{c_{p 2}^{\prime}}-\frac{c_{p 1}}{c_{p 1}^{\prime}}\right)^{2}+\left(\frac{c_{p 2}}{c_{p 2}^{\prime}}-\frac{c_{p 0}}{c_{p 0}^{\prime}}\right)^{2}+\left(\frac{c_{p 1}}{c_{p 1}^{\prime}}-\frac{c_{p 0}}{c_{p 0}^{\prime}}\right)^{2} .
$$

It is known that this estimation process is very sensitive to noise. Thus, several researchers tried to reduce the number of unknowns by fixing some of the intrinsic parameters. In [3], the skew angle and the coordinates of the principal point are fixed and the aspect ratio is supposed to be known. So, there remains only 1 parameter to estimate ${ }^{2}$.

However, especially fixing the principal point is not always corresponding to the physical reality of a zoom lens, as we stated in 2.4. We therefore generalize this approach in the next section, using generic models of the interdependence of intrinsic parameters.

\section{Pre-Calibration}

In this section, we discuss how the interdependence of the intrinsic parameters can be modeled and how this affects Kruppa's equations.

\subsection{Modeling the Interdependence of Intrinsic Parameters}

We suppose that the relation between the horizontal and vertical scale factors is given by $\alpha_{u}=\tau \alpha_{v}$, with the known and constant aspect ratio $\tau$. Furthermore, the skew angle $\theta$ is constant $\left(90^{\circ}\right)$ and hence does not depend on other parameters. As for the coordinates of the principal point, we try to model them by functional relationships on $\alpha_{v}$. Here we restrict ourselves to polynomial models, i.e.

$$
\begin{aligned}
& u_{0}\left(\alpha_{v}\right)=c_{m} \alpha_{v}^{m}+\ldots+c_{1} \alpha_{v}+c_{0} \\
& v_{0}\left(\alpha_{v}\right)=d_{n} \alpha_{v}^{n}+\ldots+d_{1} \alpha_{v}+d_{0}
\end{aligned}
$$

To determine the degrees $m$ and $n$ and the coefficients $c_{i}$ and $d_{i}$ of the models, the camera has to be calibrated by a classical method ([11]) for several zoom positions, which results in an array of parameter values (see Figure 4). From these, $c_{i}$ and $d_{i}$ can be obtained such that the model approximates well the calibration data. To obtain a sufficiently good model, but of minimal degree $m$ resp. $n$, a robust regression method ([12]) can be applied.

\subsection{How do Interdependencies affect Kruppa's Equations?}

The Kruppa equations (1) have the following form (the coefficients of the terms are not presented; these depend uniquely on the epipolar geometry $)^{3}$ :

$$
\begin{aligned}
0= & \alpha_{v}^{4}+ \\
& \alpha_{v}^{2}\left(u_{0}^{2}+v_{0}^{2}+u_{0} v_{0}+u_{0}+v_{0}+1\right)+ \\
& u_{0}^{4}+u_{0}^{3} v_{0}+u_{0}^{2} v_{0}^{2}+u_{0} v_{0}^{3}+v_{0}^{4}+u_{0}^{3}+u_{0}^{2} v_{0}+u_{0} v_{0}^{2}+v_{0}^{3}+u_{0}^{2}+\ldots+1 .
\end{aligned}
$$

\footnotetext{
${ }^{2}$ To be precise, in [3] two cameras with possibly different intrinsic parameters are considered and thus that work deals with two unknowns. However, the approach can be specialized to our context.

${ }^{3}$ For one of the 3 equations, the term $u_{0}^{4}$ vanishes, and for a second equation, $v_{0}^{4}$ vanishes. However, this does not influence further conclusions.
} 
Thus, if $u_{0}$ and $v_{0}$ are modeled as in equations (3), Kruppa's equations become polynomials in the sole parameter $\alpha_{v}$, of degree $\max \{4,4 \max \{m, n\}\}$.

In the following, we discuss first the special cases $m=n=0$ and $m=n=1$ and then the general case.

$\boldsymbol{m}=\boldsymbol{n}=\mathbf{0}$ : fixed principal point . This case has already been considered in [3]. It is interesting to note that here, Kruppa's equations have the form $a \alpha_{v}^{4}+b \alpha_{v}^{2}+c=0$. Hence, we obtain $\alpha_{v}$ by solving quadratic polynomials which leads to a closed-form solution of the problem.

$\boldsymbol{m}, \boldsymbol{n} \leq 1$ : translating principal point . Kruppa's equations are general polynomials of degree 4, i.e. also the odd coefficients may be non-zero. Hence, there exists also a closed-form solution to find possible candidates for $\alpha_{v}$.

$\boldsymbol{m}>\mathbf{1}$ or $\boldsymbol{n}>\mathbf{1}$. Kruppa's equations are of degree greater than 5. There is no analytical solution, however, since the equations are univariate polynomials, their roots can be easily computed by numerical techniques.

\section{A Method of Self-Calibration based on Pre-Calibration of the Camera}

In this section, we are interested in how self-calibration methods can be simplified and made more accurate, when an analytical expression of the interdependence of intrinsic parameters is given by pre-calibration (see previous section).

For each pair of views, there are 3 Kruppa equations (only two independent). Let $k_{p i}$ be the $i$ th Kruppa equation of the $p$ th pair of views. These equations are polynomials in the sole parameter $\alpha_{v}$ (see 4.2). Thus, $\alpha_{v}$ is the root which is common to all considered Kruppa equations. To estimate $\alpha_{v}$, we first compute the roots for each Kruppa equation separately ( $c f$. 4.2). Since the $\alpha_{v}$ must be real and positive, only the roots with these properties are retained.

Let $S_{p i}=\left\{r_{p i j} \mid j=1, \ldots, n_{p i}\right\}$ be the set of real positive roots of the Kruppa equation $k_{p i}$. We search now for the best approximation of the root which is common to all considered Kruppa equations. In this context we define the "distance" of a number $r$ to a set of numbers $S=\left\{r_{1}, \ldots, r_{n}\right\}$ as : $d(r, S)=\min _{j=1, \ldots, n}\left|r-r_{j}\right|$. Hence, the problem can be formulated as finding the real number $r$ which minimizes

$$
\sum_{p, i} d\left(r, S_{p i}\right)=\sum_{p, i} \min _{j=1, \ldots, n_{p i}}\left|r-r_{p i j}\right| .
$$

We propose a simple practical solution to this problem. Consider Figure 2 (a) which shows the graph of $d(r, S)$ for a given set $S=\left\{r_{1}, \ldots, r_{n}\right\}$.

The function is piecewise linear and changes its shape only at the abscissae $r_{j}$ and $\frac{r_{i}+r_{i+1}}{2}$. Thus, to find the global minimum of the sum $\sum_{p, i} d\left(r, S_{p i}\right)$, it is sufficient to evaluate it at all abscissae $r_{p i j}$ and $\frac{r_{p i j}+r_{p i, j+1}}{2}$ and to pick out the abscissa $r$ for which we get the minimum value. This abscissa is not uniquely defined if we have an even number of Kruppa equations ( $c f$. Figure 2 (b), where the global minimum lies between the abscissae 20 and 23). In this case, we choose the middle value.

An example of the graphs of the 3 Kruppa equations which arise from 2 views is shown in Figure 3. 

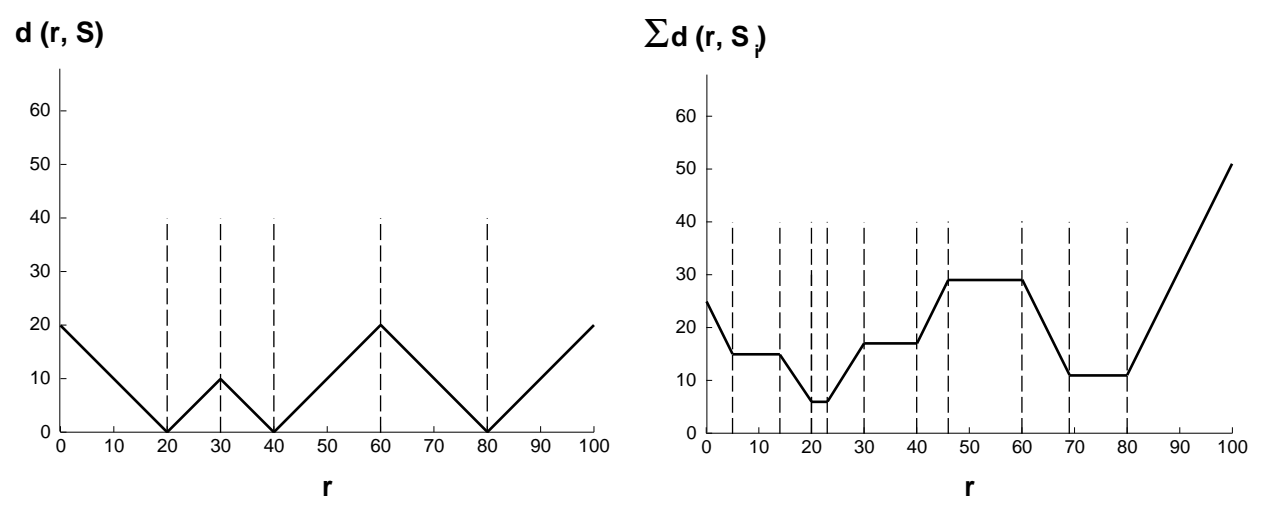

Figure 2: (a) $d(r, S)$ for $S=\{20,40,80\}$. (b) $d\left(r, S_{1}\right)+d\left(r, S_{2}\right)$ for $S_{1}=\{20,40,80\}$ and $S_{2}=\{5,23,69\}$. The dotted lines indicate the abscissa where the functions have to be evaluated to find the global minimum.

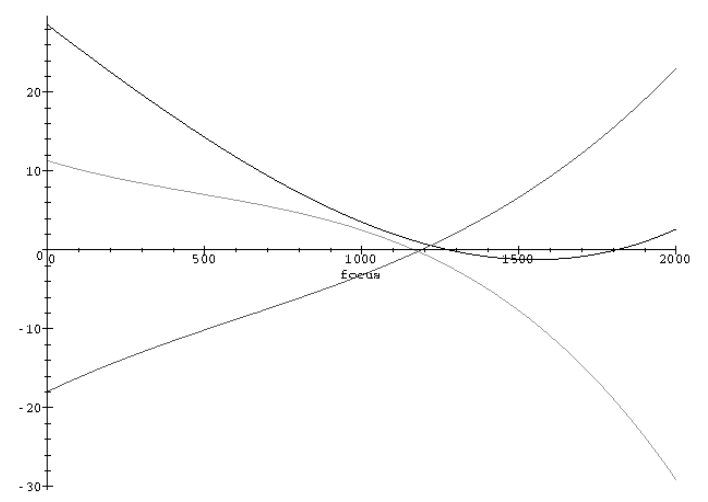

Figure 3: Example for the graphs of the 3 Kruppa equations for 2 views.

When many views are used for self-calibration, robust methods ([12]) should be applied for the estimation of the common root.

\subsection{Outline of Dynamic Self-Calibration}

0. Pre-calibrate the camera, i.e. determine the functional relationship of intrinsic parameters in dependence of $\alpha_{v}$ (see 4.1).

1. Compute the fundamental matrices for pairs of views from correspondences of image points.

2. Compute the coefficients of the powers of $\alpha_{v}$ in the Kruppa equations (see 4.2).

3. Compute the roots of each Kruppa equation and retain the real positive ones (see 5).

4. $\alpha_{\imath}$ is the "best" common root of the Kruppa equations (see 5).

5. Compute the other intrinsic parameters from $\alpha_{v}$, following the functional relationships established in step 0. 


\section{Experimental Evaluation}

We have carried out experiments with an E.I.A. Servolens zoom, mounted on a Pulnix TM-6EX camera. In a first step, the camera was pre-calibrated. For this purpose, a full off-line calibration was done for various zoom positions, by pointing the camera at a calibration grid. The field of computed intrinsic parameters is shown in Figure 4, where $u_{0}, v_{0}$ and $\alpha_{u}$ are displayed in relation to $\alpha_{v}$.
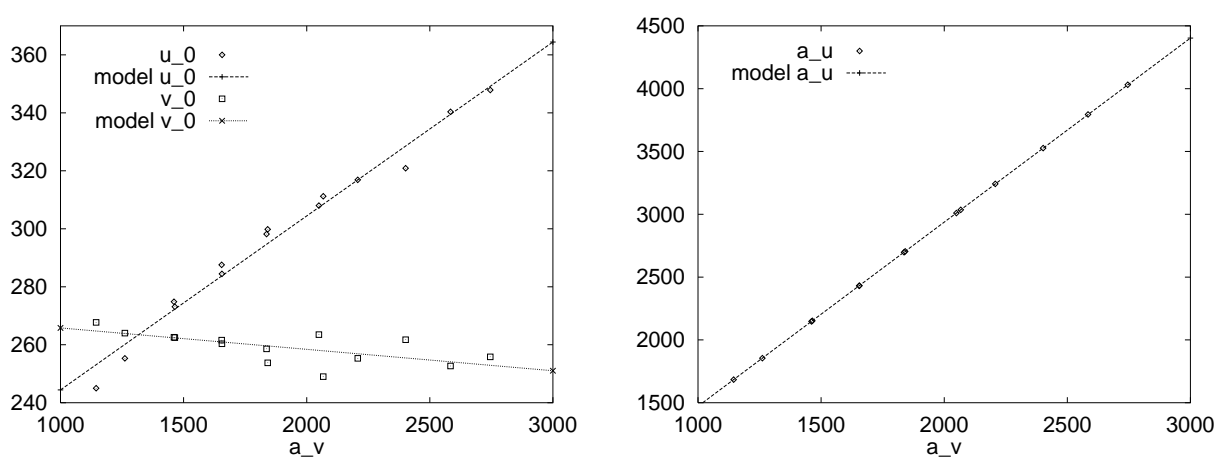

Figure 4: (a) Coordinates of the principal point $\left(u_{0}, v_{0}\right)$ with respect to $\alpha_{v}$. (b) $\alpha_{u}$ with respect to $\alpha_{v}$. The points in these graphs were obtained by classical calibration using a calibration grid ([11]). For each zoom position, several positions of the grid were used. The plotted lines represent the models found for the parameters' interdependence.

Next, the functional relationship between the intrinsic parameters was modeled. We found the following linear relations :

$$
\alpha_{u}=1.466 \alpha_{v}, u_{0}=0.060 \alpha_{v}+184.44, v_{0}=-0.007 \alpha_{v}+273.19 .
$$

The skew angle $\theta$ is fixed to $90^{\circ}$.

Dynamic self-calibration was then performed by pointing the pre-calibrated camera at the calibration grid, from different viewpoints. We compared our method with an algorithm performing a Levenberg-Marquardt (LM) minimization of the criterion (2). In contrast to our algorithm, this non-linear optimization needs an initialization of the intrinsic parameters. We applied the algorithm on 9 different initializations, which all verified exactly the model established in pre-calibration and which covered the complete range of considered zoom positions. Furthermore, the LM-algorithm was run in 4 different modes : keeping constant

- the initial values of the aspect ratio $\tau$ and $u_{0}$ and $v_{0}$.

- the initial value of the aspect ratio $\tau$.

- the initial values of $u_{0}$ and $v_{0}$.

- none of the parameters.

To summarize, one experiment consisted in applying our algorithm without initialization and running the 4 versions of the LM-method, each one with 9 different initializations. 27 different experiments were carried out: for each of 9 zoom positions, 4 images of a calibration grid were taken, and self-calibration was carried out for 2, 3 and all of 


\section{British Machine Vision Conference}

the images. The estimation of the intrinsic parameters was then evaluated based on a model-based calibration performed with the same images of the calibration grid.

In Figure 5 we summarize the results of the estimation of $\alpha_{u}$. Each bar represents the number of experiments for which the relative error of the estimated $\alpha_{u}$ lies in the corresponding error interval. The upper bars correspond to the method proposed in this paper and the lower ones to the LM-algorithm for which $\tau, u_{0}$ and $v_{0}$ were kept fixed, which has been found to be the best among the different modes.

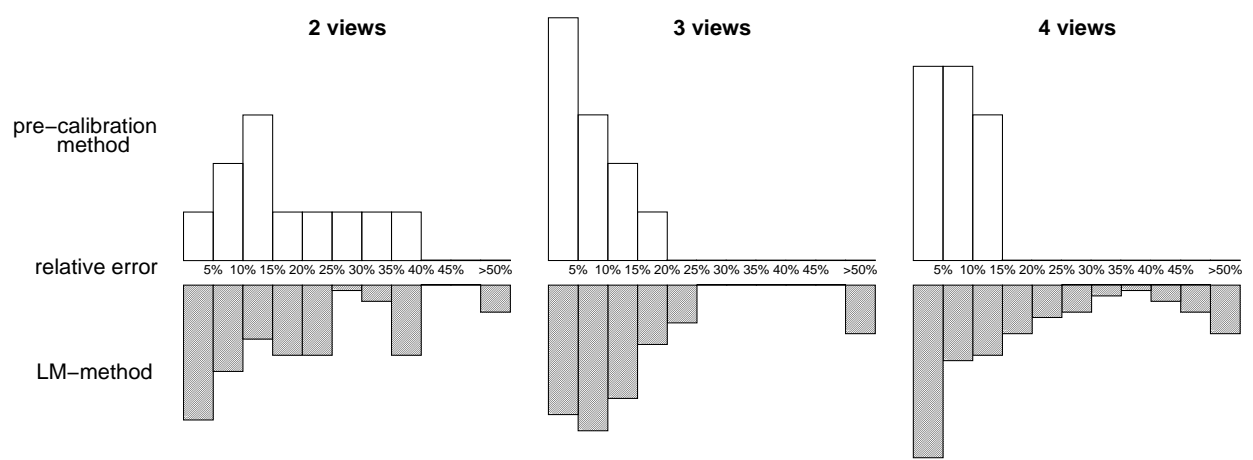

Figure 5: Results of dynamic self-calibration.

The major conclusion is that, for more than 2 views, our method was not subject to serious failure, contrary to the LM-method, which completely failed in more than $5 \%$ of the experiments. However, when the initialization was close to the true values, the LM-method gave also mostly acceptable results.

The graphs for estimation of $u_{0}$ and $v_{0}$ (not shown here) underline the higher reliability of our method with respect to self-calibration which does not use all available pre-calibration information.

\section{Conclusions and Further Work}

We have examined the interdependence of intrinsic parameters of a zooming camera in order to reduce the number of unknowns in self-calibration processes. Especially we have shown that, when the pinhole model is assumed, self-calibration consists in the estimation of only 1 intrinsic parameter. We propose a special algorithm which exploits the interdependence of the parameters and which is based on an off-line pre-calibration of the camera. The algorithm needs only simple computations (roots of univariate polynomials) and furthermore, no initialization of the parameters is necessary. Experiments have shown, that the proposed method is more reliable than classical non-linear optimization which does not exploit completely the interdependence of the intrinsic parameters.

The major drawback is of course the need of a time-consuming pre-calibration process. It is also not clear if the proposed mechanism will be very effective for imaging systems where the interdependence of parameters follows a more complicated model as the one found with our equipment.

In this paper we have neglected the effect of focusing. This could be dealt with by doing pre-calibration for different focus settings and establishing different interdependence 


\section{British Machine Vision Conference}

models which then cover the whole range of focus and zoom settings. The method could then be run for these different models and the self-calibration which gives the lowest re-projection residue for the reconstruction of the observed scene, would be retained.

The actual implementation of the algorithm could be extended in several directions : The search for the common root of many Kruppa equations can be done by a robust method. Uncertainty estimates for the fundamental matrices can be taken into account when many views are given. On the experimental side, experiments with more than 4 images and with real images (not a calibration grid) should be undertaken.

Acknowledgements This work was partially supported by INRIA France. We thank Reg Willson for the kind permission to publish Figure 1, Philippe Bobet for helpful discussions and Pascal Brand for his help for carrying out the experiments.

\section{References}

[1] Maybank, S J, Faugeras, O D, "A Theory of Self Calibration of a Moving Camera", International Journal of Computer Vision, 8, 2, 1992, pp 123-151.

[2] Luong, Q-T, Matrice fondamentale et autocalibration en vision par ordinateur, Thèse de doctorat, Université de Paris-Sud, Orsay, France, Dec 1992.

[3] Hartley, R, Extraction of Focal Lengths from the Fundamental Matrix, G.E. CRD, Schenectady, NY, 1993.

[4] Enciso, R, Viéville, T, "Experimently Self-Calibration from Four Views”, Proc. 1995 Int. Conf. on Image Analysis and Processing, San Remo, Italy, Sept 1995.

[5] Willson, R G, Shafer, S A, "A Perspective Projection Camera Model for Zoom Lenses", Proc. 2nd Conf. on Optical 3-D Measurement Techniques, Zürich, Switzerland, Oct 1993.

[6] Burner, A W, “Zoom Lens Calibration for Wind Tunnel Measurements”, Proc. SPIE Conf. on Videometrics IV, Philadelphia, PA, Oct 1995, pp 19-33.

[7] Semple, J G, Kneebone, G T, Algebraic Projective Geometry, Oxford Science Publication, 1952.

[8] Hartley, R I, "Euclidean Reconstruction from Uncalibrated Views", Proc. DARPAESPRIT Workshop on Applications of Invariants in Computer Vision, Azores, Portugal, Oct 1993, pp 187-202.

[9] Brown, D C, "Close-Range Camera Calibration", Photogrammetric Engineering, 37, 8, 1971, pp 855-866.

[10] Faugeras, O, "What Can Be Seen in Three Dimensions with An Uncalibrated Stereo Rig ?", Proc. 2nd ECCV, Santa Margherita, Italy, May 992, 563-578.

[11] Faugeras, O D, Toscani, G, "Camera Calibration for 3D Computer Vision”, Proc. Int. Workshop Machine Vision and Machine Intelligence, Tokyo, 1987.

[12] Meer, P, Mintz, D, Rosenfeld, A, Kim, D Y, "Robust Regression Methods for Computer Vision: A Review”, Int. Jrnl. Comp. Vision, 6, 1, 1991, pp 59-70. 